\title{
Multi-elemental analysis of low-volume samples reveals cancer-specific profile in serum and sorted immune cells
}

\author{
- Supporting Information -
}

Tobias Konz ${ }^{1, *}$, Caroline Monnard ${ }^{1}$, Marcela Rincon Restrepo ${ }^{2}$, Julie Laval ${ }^{1}$, Federico Sizzano ${ }^{3}$, Mukul Girotra ${ }^{2}$, Gabriele Dammone ${ }^{3}$, Alessio Palini ${ }^{3}$, George Coukos ${ }^{4}$, Serge Rezzi ${ }^{1, a}$, Jean-Philippe Godin ${ }^{1, *}$ and Nicola Vannini2,*

\footnotetext{
${ }^{1}$ Nestlé Research, Vers-chez-les-Blanc, Lausanne, Switzerland

${ }^{2}$ Laboratory of Immunosenscence and Stem Cell Metabolism, Department of Oncology UNIL CHUV, Ludwig Institute for Cancer Research Lausanne, University of Lausanne, Epalinges 1066, Switzerland ${ }^{3}$ Nestlé Research, EPFL Innovation Park, Lausanne, Switzerland

${ }^{4}$ Human Integrated Tumor Immunology Discovery Engine, Department of Oncology UNIL CHUV, Ludwig Institute for Cancer Research Lausanne, University of Lausanne, Epalinges 1066, Switzerland
}

${ }^{a}$ Current address: Swiss Vitamin Institute, Epalinges, Switzerland

\section{$\underline{\text { Table of content }}$}

\begin{tabular}{|c|c|c|}
\hline Page & Content & Title \\
\hline S-2 & Table S1 & Elemental calibration ranges applied for the serum and cell method. \\
\hline S-2 & Table S2 & Experimental conditions of the ICP-MS/MS system. \\
\hline $\mathrm{S}-3$ & Table S3 & Summary of the acquisition method ( $\mathrm{He}$ - and $\mathrm{O}_{2}$-gas mode) using ICP-MS. \\
\hline $\mathrm{S}-3$ & Table S4 & Peristaltic pump program for the ICP-MS. \\
\hline S-4 & Table S5 & Calculated LOD, LOQ and corresponding elemental transition \\
\hline $\mathrm{S}-5$ & Table S6 & Summary of analytical figures of merit of the proposed method. \\
\hline S-7 & Figure S1 & $\begin{array}{l}\text { Recovery, (expressed in \%), of T cells sorted in different collection buffers } \\
\text { at different BSA concentrations. }\end{array}$ \\
\hline S-7 & Figure S2 & Elemental concentrations in standard PBS buffer, expressed in $\mathrm{ng} \mathrm{mL}^{-1}$. \\
\hline S-8 & Table S7 & $\begin{array}{l}\text { Concentration ranges ( } \mathrm{ng} \mathrm{mL} \mathrm{mL}^{-1} \text { ) in serum of naïve (control) and tumor- } \\
\text { bearing mice (cancer MC38 and Cancer B16). Median + IR [25; 75\%]. }\end{array}$ \\
\hline S-8 & Table S8 & T cell concentration ranges (fg cell ${ }^{-1}$ expressed as Median + IR [25;75\%]). \\
\hline S-9 & Figure S3 & $\begin{array}{l}\text { Comparison of elemental concentrations in } \mathrm{T} \text { cells purified by FACS } \\
\text { sorting and beads precipitation. }\end{array}$ \\
\hline
\end{tabular}


Table S1: Elemental calibration ranges applied for the serum and cell method. For external calibration, two different sets of calibrants were prepared in order to avoid cross contamination and to cope with the huge concentration differences between elements under evaluation. Set 1 consisting of $\mathrm{Mg}, \mathrm{P}, \mathrm{S}, \mathrm{K}, \mathrm{Ca}$ and $\mathrm{Mo}$ and set 2 containing $\mathrm{V}, \mathrm{Cr}, \mathrm{Mn}, \mathrm{Fe}, \mathrm{Co}, \mathrm{Cu}, \mathrm{Zn}, \mathrm{Se}, \mathrm{Br}, \mathrm{Rb}, \mathrm{Sr}, \mathrm{I}, \mathrm{Cs}$ and $\mathrm{Ba}$. The final calibration ranges where obtained as follows: For serum, we have evaluated the reported reference ranges for each element. The calibration ranges for serum were adapted accordingly, ensuring that each calibration range covers the expected concentration range in the sample. For the cell method we have studied the concentration ranges of elements in different cell types (e.g. immune cells, IPSCs) that we analyzed during this project. We observed relatively large concentration differences among the elements under evaluation. Therefore, the concentration ranges for the cell method were adapted to the cell population under evaluation.

\begin{tabular}{|l|r|r|}
\hline \multirow{2}{*}{ Element } & \multicolumn{1}{|c|}{ Serum } & \multicolumn{1}{|c|}{ Cells } \\
\cline { 2 - 3 } & \multicolumn{2}{|c|}{$\mathrm{ng} \mathrm{mL}^{-1}$} \\
\hline $\mathrm{Mg}$ & $37-6000$ & $1-150$ \\
\hline $\mathrm{P}$ & $123-20000$ & - \\
\hline $\mathrm{S}$ & $1841-300000$ & $15-2500$ \\
\hline $\mathrm{Ca}$ & $172-28000$ & - \\
\hline $\mathrm{K}$ & $540-88000$ & - \\
\hline $\mathrm{V}$ & $0.0002-0.2$ & - \\
\hline $\mathrm{Cr}$ & $0.001-0.8$ & $0.003-2.4$ \\
\hline $\mathrm{Mn}$ & $0.003-3.2$ & $0.04-36$ \\
\hline $\mathrm{Fe}$ & $0.4-900$ & $0.001-0.075$ \\
\hline $\mathrm{Co}$ & $0.0005-0.5$ & $0.001-1.2$ \\
\hline $\mathrm{Cu}$ & $0.5-990$ & $0.01-12$ \\
\hline $\mathrm{Zn}$ & $0.4-900$ & $0.004-0.36$ \\
\hline $\mathrm{Se}$ & $0.04-90$ & -240 \\
\hline $\mathrm{Br}$ & $1-2070$ & - \\
\hline $\mathrm{Sr}$ & $0.1-150$ & \\
\hline $\mathrm{Rb}$ & $0.1-160$ & $0.002-1.44$ \\
\hline $\mathrm{Mo}$ & $0.01-1$ & $0.0005-0.076$ \\
\hline $\mathrm{I}$ & $0.04-80$ & - \\
\hline $\mathrm{Cs}$ & $0.004-3.75$ & $0.0001-0.075$ \\
\hline $\mathrm{Ba}$ & $0.02-45$ & - \\
\hline
\end{tabular}

Table S2. Experimental conditions of the ICP-MS/MS system.

\begin{tabular}{|c|c|}
\hline Parameter & Value \\
\hline Plasma mode & Low matrix \\
\hline $\mathrm{RF}^{*}$ power $[\mathrm{W}]$ & 1550 \\
\hline Plasma gas $\left(\mathrm{L} \mathrm{min} \mathrm{min}^{-1}\right)$ & 15 \\
\hline Auxiliary gas (L min $\left.{ }^{-1}\right)$ & 0.9 \\
\hline Carrier gas $\left(\mathrm{L} \mathrm{min}^{-1}\right)$ & 1.09 \\
\hline Sampler and skimmer cones & Nickel \\
\hline Spray chamber & Scott double pass \\
\hline Nebulizer & Burgener MicroMist \\
\hline Peristaltic pump & $0.1 \mathrm{rps}$ \\
\hline Cell gas $(\mathrm{He})$ & $5 \mathrm{ml} \mathrm{min}^{-1}$ \\
\hline Cell gas $\left(\mathrm{O}_{2}\right)$ & $30 \%$ \\
\hline
\end{tabular}

*RF: radio frequency; rps: rotations per second 
Table S3: Summary of the acquisition method (He- and $\mathrm{O}_{2}$-gas mode) using ICP-MS.

\begin{tabular}{|c|c|c|c|c|}
\hline Element Name & Mass & $\begin{array}{c}\text { Integration Time } \\
\text { /Mass } \\
\text { [sec] }\end{array}$ & $\mathrm{Q} 1->\mathrm{Q} 2$ & $\begin{array}{c}\text { Integration Time } \\
\text { /Mass } \\
{[\mathrm{sec}]}\end{array}$ \\
\hline Tune mode & \multicolumn{2}{|r|}{$\mathrm{He}$} & \multicolumn{2}{|r|}{$\mathrm{O}_{2}$} \\
\hline $\mathrm{Mg}$ & 24 & 0.1 & & \\
\hline$P$ & & & $31->47$ & 0.5 \\
\hline $\mathrm{S}$ & & & $32->48$ & 0.5 \\
\hline $\mathrm{K}$ & 39 & 0.1 & & \\
\hline $\mathrm{Ca}$ & 44 & 0.5 & & \\
\hline Sc (internal standard) & 45 & 0.1 & $45->61$ & 0.1 \\
\hline $\mathrm{V}$ & 51 & 2 & & \\
\hline $\mathrm{Cr}$ & & & $52->68$ & 2 \\
\hline $\mathrm{Mn}$ & 55 & 1 & & \\
\hline $\mathrm{Fe}$ & 56 & 1 & & \\
\hline Co & 59 & 3 & & \\
\hline $\mathrm{Cu}$ & 63 & 2 & & \\
\hline $\mathrm{Zn}$ & 66 & 1 & & \\
\hline Ga (internal standard) & 71 & 0.1 & $71->71$ & 0.1 \\
\hline $\mathrm{Br}$ & 79 & 2 & & \\
\hline Se & & & $80->96$ & 3 \\
\hline $\mathrm{Rb}$ & 85 & 1 & & \\
\hline $\mathrm{Sr}$ & 88 & 1 & & \\
\hline Mo & 98 & 3 & & \\
\hline In (internal standard) & 115 & 0.1 & $115->115$ & 0.1 \\
\hline Sn (for internal standard correction) & 118 & 0.3 & $118->118$ & 0.1 \\
\hline Te (internal standard) & 125 & 0.1 & $125->125$ & 0.1 \\
\hline 1 & 127 & 3 & & \\
\hline Cs & 133 & 2 & & \\
\hline $\mathrm{Ba}$ & 138 & 1 & & \\
\hline Bi (internal standard) & 209 & 0.1 & & \\
\hline
\end{tabular}

Of note, concentration applied for the internal standard (ISTD) is typical for ICP-MS analysis when working with online ISTD. The solution containing the single element standards (online ISTD) is mixed with the sample before reaching the nebulizer. The ratio between sample and online ISTD is roughly 90:10, meaning that the concentration of the ISTD reaching the plasma is much lower than in the online-ISTD solution. We have selected the elements beryllium, scandium, gallium, indium, tellurium and bismuth with low, medium and high $\mathrm{m} / \mathrm{z}$ ratios. Moreover, these elements are not present in the biological specimens.

Table S4: Peristaltic pump program for the ICP-MS.

\begin{tabular}{|r|c|c|c|}
\cline { 3 - 4 } \multicolumn{2}{c|}{} & $\begin{array}{c}\text { Time } \\
{[\mathrm{sec}]}\end{array}$ & $\begin{array}{c}\text { Speed peripump } \\
{[\mathrm{rps}]}\end{array}$ \\
\hline \multirow{2}{*}{ Pre-run } & Sample uptake & 80 & 0.3 \\
\cline { 2 - 4 } & Stabilize & 30 & Tune parameters \\
\hline Acquisition & Speed & & Tune parameters \\
\hline \multirow{3}{*}{ Post run } & Probe rinse (Sample) & 10 & 0.3 \\
\cline { 2 - 4 } & Probe rinse (Standard) & 10 & 0.3 \\
\cline { 2 - 4 } & Rinse 1 & 15 & \\
\cline { 2 - 4 } & Preemptive rinse & 120 & \\
\hline
\end{tabular}


Table S5. Calculated LOD, LOQ and corresponding elemental transitions.

\begin{tabular}{|l|l|l|l|l|}
\hline Element & Transition & $\begin{array}{c}\text { LOD } \\
{\left[\mathrm{ng} \mathrm{mL}^{-1}\right]}\end{array}$ & $\begin{array}{c}\text { LOQ } \\
{\left[\mathrm{ng} \mathrm{mL}^{-1}\right]}\end{array}$ & $\begin{array}{c}\text { Required } \\
\text { LOQ* } \\
{\left[\mathrm{ng} \mathrm{mL}^{-1}\right.}\end{array}$ \\
\hline Magnesium & $24 \mathrm{Mg}[\mathrm{He}]$ & 0.15 & 0.44 & 1820 \\
\hline Phosphorous & $31 \rightarrow 47 \mathrm{P}\left[\mathrm{O}_{2}\right]$ & 1.09 & 3.31 & 6300 \\
\hline Sulfur & $32 \rightarrow 48 \mathrm{~S}\left[\mathrm{O}_{2}\right]$ & 4.88 & 13.67 & 103320 \\
\hline Potassium & $39 \mathrm{~K}[\mathrm{He}]$ & 1.25 & 3.78 & 12600 \\
\hline Calcium & $43 \mathrm{Ca}[\mathrm{He}]$ & 22.3 & 67.5 & 8817 \\
\hline Vanadium & $51 \rightarrow 67 \mathrm{~V}\left[\mathrm{O}_{2}\right]$ & 0.001 & 0.004 & 0.0015 \\
\hline Chromium & $52 \rightarrow 68 \mathrm{Cr}\left[\mathrm{O}_{2}\right]$ & 0.017 & 0.050 & 0.008 \\
\hline Manganese & $55 \mathrm{Mn}[\mathrm{He}]$ & 0.012 & 0.038 & 0.03 \\
\hline Iron & $56 \mathrm{Fe}[\mathrm{He}]$ & 0.072 & 0.219 & 55 \\
\hline Cobalt & $59 \mathrm{Co}[\mathrm{He}]$ & $<0.001$ & 0.001 & 0.003 \\
\hline Copper & $63 \mathrm{Cu}[\mathrm{He}]$ & 0.029 & 0.087 & 74 \\
\hline Zinc & $66 \mathrm{Zn}[\mathrm{He}]$ & 0.080 & 0.241 & 42 \\
\hline Selenium & $80 \rightarrow 96 \mathrm{Se}\left[\mathrm{O}_{2}\right]$ & 0.018 & 0.054 & 7.4 \\
\hline Bromine & $79 \mathrm{Br}[\mathrm{He}]$ & 0.192 & 0.582 & 120 \\
\hline Rubidium & $85 \mathrm{Rb}[\mathrm{He}]$ & 0.002 & 0.005 & 1.313 \\
\hline Strontium & $88 \mathrm{Sr}[\mathrm{He}]$ & 0.042 & 0.126 & 1.2 \\
\hline Molybdenum & $98 \mathrm{Mo}[\mathrm{He}]$ & 0.001 & 0.002 & 0.027 \\
\hline lodine & $127 \mathrm{I}[\mathrm{He}]$ & 0.055 & 0.167 & 6.5 \\
\hline Cesium & $133 \mathrm{Cs}[\mathrm{He}]$ & 0.000 & 0.001 & 0.05 \\
\hline Barium & $138 \rightarrow 154 \mathrm{Ba}\left[\mathrm{O}_{2}\right]$ & 0.006 & 0.017 & 0.04 \\
\hline
\end{tabular}

LOD: limits of detection; LOQ: limits of quantification; LODs and LOQs were calculated by dividing the standard deviation of the response by the slope of the detector response, multiplied by 3 and 10 , respectively;

*required LOQ taken from [24]. 
Table S6. Summary of analytical figures of merit of the proposed method. Analysis of human serum certified reference material was performed in quintuplicate on six days. Reference values and median of the results are expressed as $\mathrm{ng} \mathrm{mL}^{-1}$, trueness, precision (repeatability and intermediate reproducibility) and measurement uncertainty are expressed in percentages (\%).

\begin{tabular}{|c|c|c|c|c|c|c|}
\hline \multirow[t]{2}{*}{ Analyte } & \multirow{2}{*}{$\begin{array}{l}\text { Ref. value } \\
{\left[\mathrm{ng} \mathrm{mL} \mathrm{mL}^{-1}\right]}\end{array}$} & \multirow{2}{*}{$\begin{array}{l}\text { Median } \\
\text { of results } \\
{\left[\mathrm{ng} \mathrm{mL}^{-1}\right]}\end{array}$} & \multirow{2}{*}{$\begin{array}{c}\text { Trueness } \\
{[\%]} \\
\end{array}$} & $\begin{array}{c}\text { Repeat } \\
\text {. }\end{array}$ & $\begin{array}{l}\text { Inter. } \\
\text { Repr. }\end{array}$ & $\begin{array}{c}\text { Relative } \\
\text { uncertaint } \\
y\end{array}$ \\
\hline & & & & $\begin{array}{c}\mathrm{CV}(\mathrm{r}) \\
{[\%]}\end{array}$ & $\begin{array}{c}\mathrm{CV}(\mathrm{iR}) \\
{[\%]}\end{array}$ & $\begin{array}{c}\text { Rel. u } \\
{[\%]}\end{array}$ \\
\hline $\mathrm{Mg}$ & 33900 & 33095 & 97.6 & 2.1 & 8.7 & 18.7 \\
\hline $\mathrm{P}$ & 110000 & 112670 & 102.4 & 2.5 & 5.6 & 12.0 \\
\hline$S$ & 1335000 & 1411176 & 105.7 & 2.7 & 5.6 & 11.9 \\
\hline $\mathrm{K}$ & 221000 & 229826 & 104.0 & 2.1 & 10.2 & 21.9 \\
\hline $\mathrm{Ca}$ & 119000 & 112859 & 94.8 & 1.5 & 12.8 & 27.5 \\
\hline $\mathrm{V}$ & 1.10 & 1.04 & 94.4 & 2.7 & 9.7 & 20.8 \\
\hline $\mathrm{Cr}$ & 5.70 & 4.97 & 87.2 & 4.9 & 5.1 & 16.7 \\
\hline $\mathrm{Mn}$ & 14.50 & 13.32 & 91.9 & 2.3 & 15.6 & 33.8 \\
\hline $\mathrm{Fe}$ & 2150 & 2089 & 97.2 & 2.3 & 10.3 & 22.3 \\
\hline Co & 3.05 & 3.03 & 99.4 & 2.6 & 13.5 & 29.2 \\
\hline $\mathrm{Cu}$ & 1850 & 1966 & 106.3 & 2.2 & 11.8 & 25.4 \\
\hline $\mathrm{Zn}$ & 1617 & 1420 & 87.8 & 1.8 & 16.0 & 34.6 \\
\hline $\mathrm{Se}$ & 138.0 & 129.57 & 93.9 & 2.0 & 13.5 & 29.1 \\
\hline $\mathrm{Br}$ & 773.0 & 689.94 & 89.3 & 2.2 & 14.8 & 31.9 \\
\hline $\mathrm{Rb}$ & 8.70 & 8.35 & 96.0 & 2.1 & 8.0 & 17.2 \\
\hline $\mathrm{Sr}$ & 110.0 & 105.27 & 95.7 & 1.8 & 17.7 & 38.2 \\
\hline Mo & 1.21 & 1.08 & 89.3 & 2.9 & 13.1 & 28.2 \\
\hline 1 & 60.9 & 60.25 & 98.9 & 3.7 & 10.5 & 22.6 \\
\hline Cs & 0.026 & 0.03 & 99.1 & 13.5 & 18.0 & 37.6 \\
\hline $\mathrm{Ba}$ & 135.0 & 135.67 & 100.5 & 1.7 & 12.5 & 27.1 \\
\hline
\end{tabular}

$\mathrm{CV}(\mathrm{r})$ ): coefficient of variation (repeatability); $\mathrm{CV}(\mathrm{iR})$ : coefficient of variation of intermediate reproducibility;

Rep.: repeatability; Inter. Rep.: intermediate reproducibility; Rel. u: relative uncertainty.

$\mathrm{CV}(r)$ is the intra-day coefficient of variation meaning the standard deviation divided by the median.

$\mathrm{CV}$ (ir) is the inter-day coefficient of variation meaning the standard deviation divided by the median (measured through 6 days).

Relative uncertainty $(\mathrm{u})$ is calculated with the following formula taking into account the precision and the trueness of the method at each level of concentration to obtain the overall uncertainty (relative, expressed in percentage).

$\mathrm{u}(\%)=\sqrt{C V(i R) 2+C V(\operatorname{Rec})^{2}}$; where $\mathrm{CV}(\mathrm{iR})^{2}$ is the contribution of the precision to the uncertainty and $\mathrm{CV}(\operatorname{Rec}) 2$ is the contribution of the trueness.

$$
\begin{aligned}
& \mathrm{SD}_{\text {rob }}(\mathrm{r})=1.0484 \times \operatorname{Med}_{\mathrm{i}=1}\left\{\mathrm{x}_{\mathrm{i} 1}-\mathrm{x}_{\mathrm{i} 2} \mid\right\}_{\text {圆 }} \\
& \mathrm{SD}_{\text {rob }}(\mathrm{iR})=\sqrt{\mathrm{SD}_{\text {rob }}^{2}(\mathrm{~b})+\frac{1}{2} \times \mathrm{SD}_{\text {rob }}^{2}(\mathrm{r})}
\end{aligned}
$$

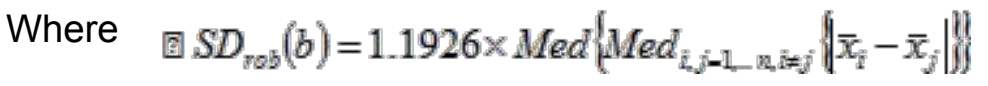

And $\quad \bar{x}_{i}$ is the average of the ith duplicate 


$$
\begin{gathered}
C V_{\text {rob }}(r)=\frac{S D(r)}{\text { Median }} \\
\mathrm{U} \%=2^{*} \mathrm{u} \% \\
\mathrm{u} \%=\sqrt{\mathrm{CV}(\mathrm{iR})^{2}+\mathrm{C} V(\operatorname{Rec})_{\text {corrected }}^{2}}
\end{gathered}
$$

If recovery is NOT statistically different from $100 \%$ :

$$
\begin{aligned}
\mathrm{SD}(\operatorname{Re} c)_{\text {corrected }} & =\mathrm{SD}(\text { Re cov ery }) \\
C V(\operatorname{Re} c)_{\text {corrected }} & =\frac{S D(\operatorname{Recov} \text { ery })}{\operatorname{Recov} \text { ery }}
\end{aligned}
$$

If recovery is different from $100 \%$ :

$$
\begin{gathered}
\operatorname{SD}(\operatorname{Rec})_{\text {corrected }}^{*}=\sqrt{\left(\frac{1-\operatorname{Recov} \text { ery }}{2}\right)^{2}+\mathrm{SD}(\operatorname{Re~cov~ery~})^{2}} \\
C V(\operatorname{Re} c)_{\text {corrected }}^{*}=\frac{\sqrt{\left(\frac{1-\operatorname{Recov} \text { ery }}{2}\right)^{2}+S D(\operatorname{Recov} \text { ery })^{2}}}{\text { Recovery }}
\end{gathered}
$$




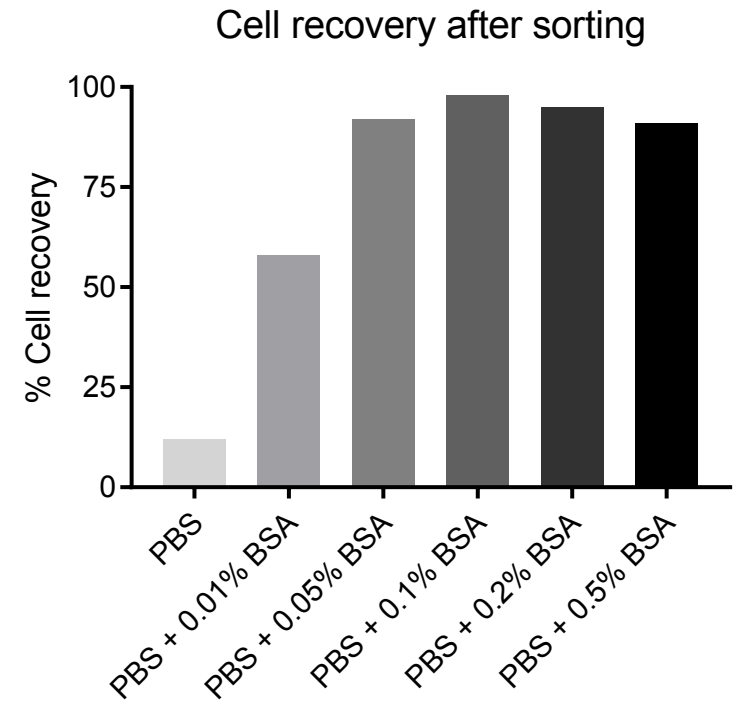

Figure S1: Recovery, (expressed in \%), of T cells sorted in different collection buffers at different BSA concentrations. Overall, there is a high consistency between the different BSA concentrations, especially at BSA concentrations $<0.05 \%$ where we observed cell recoveries of $<90 \%$. Indeed, our analyses revealed a good accordance between BSA concentrations and cell recovery capacity, where, as expected, highest concentrations reached maximal recovery efficiency (plateau). Due to the good correlations between BSA doses and curve fitting, we didn't perform replicates being not an experiment directly influencing our methodology and, for this reason, we have now moved this figure into the supporting information section.

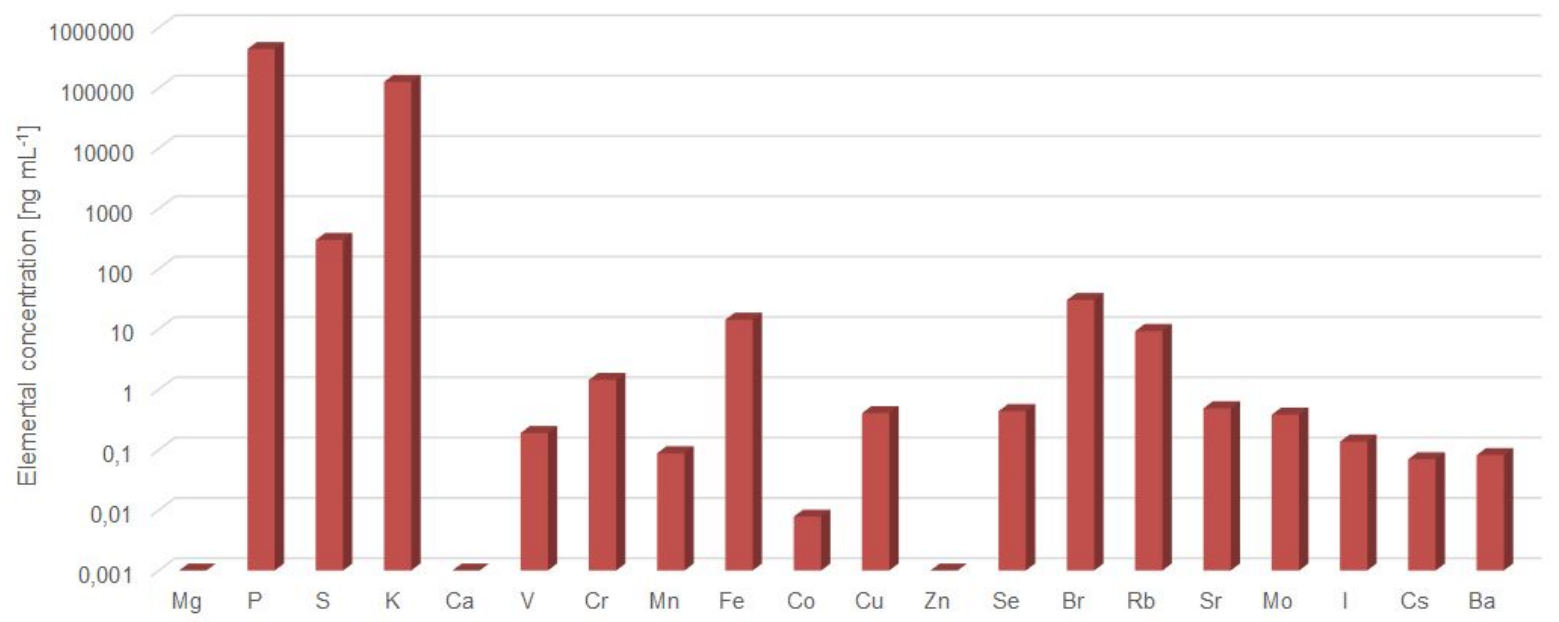

Figure S2) Elemental concentrations in standard PBS buffer, expressed in $\mathrm{ng} \mathrm{mL}^{-1}$. 
Table S7: Concentration ranges ( $\mathrm{ng} \mathrm{mL}^{-1}$ ) in serum of naïve (control) and tumor-bearing mice (cancer MC38 and Cancer B16). Median + IR [25; 75\%].

\begin{tabular}{|l|c|c|c|}
\hline Element & Control & Cancer MC38 & Cancer B16 \\
\hline Magnesium & $33634[28273 ; 41360]$ & $35597[32082 ; 38682]$ & $29464[26396 ; 32140]$ \\
Phosphorous & $151685[134188 ; 162554]$ & $165429[149328 ; 185606]$ & $181307[171552 ; 195819]$ \\
Sulfur & $923898[837395 ; 1008391]$ & $904864[864246 ; 963199]$ & $878099[858133 ; 912149]$ \\
Potassium & $217322[197078 ; 247374]$ & $205453[187337 ; 225133]$ & $249236[207687 ; 279702]$ \\
Calcium & $84728[70742 ; 97539]$ & $92460[88908 ; 99458]$ & $95134[93400 ; 96351]$ \\
Vanadium & $2.514[2.149 ; 3.071]$ & $2.423[2.303 ; 2.712]$ & $1.449[1.284 ; 1.731]$ \\
Chromium & $0.931[0.784 ; 1.326]$ & $1.186[0.867 ; 1.641]$ & $0.334[0.273 ; 0.511]$ \\
Manganese & $3.2[2.048 ; 3.578]$ & $3.255[2.883 ; 3.737]$ & $4.645[2.477 ; 5.825]$ \\
Iron & $5349[3670 ; 8115]$ & $3218[2322 ; 4836]$ & $3479[1980 ; 4557]$ \\
Cobalt & $4.8[4.017 ; 5.086]$ & $3.537[3.087 ; 3.827]$ & $5.174[3.87 ; 6.346]$ \\
Copper & $463.4[440.8 ; 502.4]$ & $516.6[498.3 ; 587]$ & $620.1[581.9 ; 701.6]$ \\
Zinc & $790.8[717.4 ; 916.9]$ & $843.9[770.9 ; 913.1]$ & $890.8[781.6 ; 947.4]$ \\
Selenium & $380.5[331 ; 399]$ & $330[313.6 ; 363.8]$ & $401[374.4 ; 428.2]$ \\
Bromine & $2388[2360 ; 2460]$ & $2215[2150 ; 2314]$ & $2892[2506 ; 3335]$ \\
Rubidium & $132.1[109.6 ; 172.6]$ & $93.53[86.04 ; 114.8]$ & $175.7[159.9 ; 223.1]$ \\
Strontium & $17.9[16.78 ; 18.24]$ & $17.5[15.51 ; 20.69]$ & $16.49[13.51 ; 17.99]$ \\
Molybdenum & $6.883[6.595 ; 9.2]$ & $7.355[6.374 ; 8.075]$ & $6.288[5.418 ; 8.127]$ \\
lodine & $118.2[113.3 ; 124.3]$ & $101.9[95 ; 116.8]$ & $87.51[80.27 ; 146.1]$ \\
Cesium & $0.31[0.242 ; 0.386]$ & $0.216[0.189 ; 0.24]$ & $0.403[0.272 ; 0.453]$ \\
Barium & $1.076[0.651 ; 1.251]$ & $1.008[0.865 ; 1.205]$ & $1.286[1.108 ; 1.441]$ \\
\hline
\end{tabular}

Table S8: T cell concentration ranges (fg cell ${ }^{-1}$ expressed as Median + IR [25;75\%]).

\begin{tabular}{|l|c|c|c|}
\hline Element & Naïve CD4 & Naïve CD8 & Cancer CD8 \\
\hline Magnesium & $29.65[28.59 ; 32.06]$ & $30.79[30.06 ; 31.54]$ & $35.66[35.18 ; 37.22]$ \\
Sulfur & $102.3[76.9 ; 154.9]$ & $104.1[86.61 ; 156.4]$ & $112.5[98.49 ; 127.8]$ \\
Manganese & $0.015[0.006 ; 0.064]$ & $0.021[0.007 ; 0.024]$ & $0.061[0.042 ; 0.1]$ \\
Iron & $0.02[0.01 ; 0.636]$ & $0.481[0.251 ; 1.499]$ & $0.4[0.299 ; 1.05]$ \\
Cobalt & $0.001[0.001 ; 0.004]$ & $0.002[0.001 ; 0.006]$ & $0.001[0.001 ; 0.002]$ \\
Copper & $0.025[0.01 ; 0.222]$ & $0.07[0.028 ; 0.095]$ & $0.138[0.067 ; 0.435]$ \\
Zinc & $4.131[1.219 ; 7.185]$ & $2.774[0.896 ; 5.704]$ & $5.840[4.711 ; 6.337]$ \\
Selenium & $0.008[0.007 ; 0.009]$ & $0.01[0.008 ; 0.014]$ & $0.01[0.007 ; 0.012]$ \\
Bromine & $2.482[1.43 ; 4.783]$ & $1.538[0.984 ; 2.219]$ & $3.148[2.055 ; 4.241]$ \\
Rubidium & $0.183[0.165 ; 0.216]$ & $0.172[0.151 ; 0.184]$ & $0.129[0.106 ; 0.162]$ \\
Molybdenum & $0.0007[0.0006 ; 0.0049]$ & $0.0012[0.0002 ; 0.0024]$ & $0.0046[0.0015 ; 0.0094]$ \\
Cesium & $0.001[0 ; 0.001]$ & $0[0 ; 0.001]$ & $0[0 ; 0.001]$ \\
\hline
\end{tabular}


A

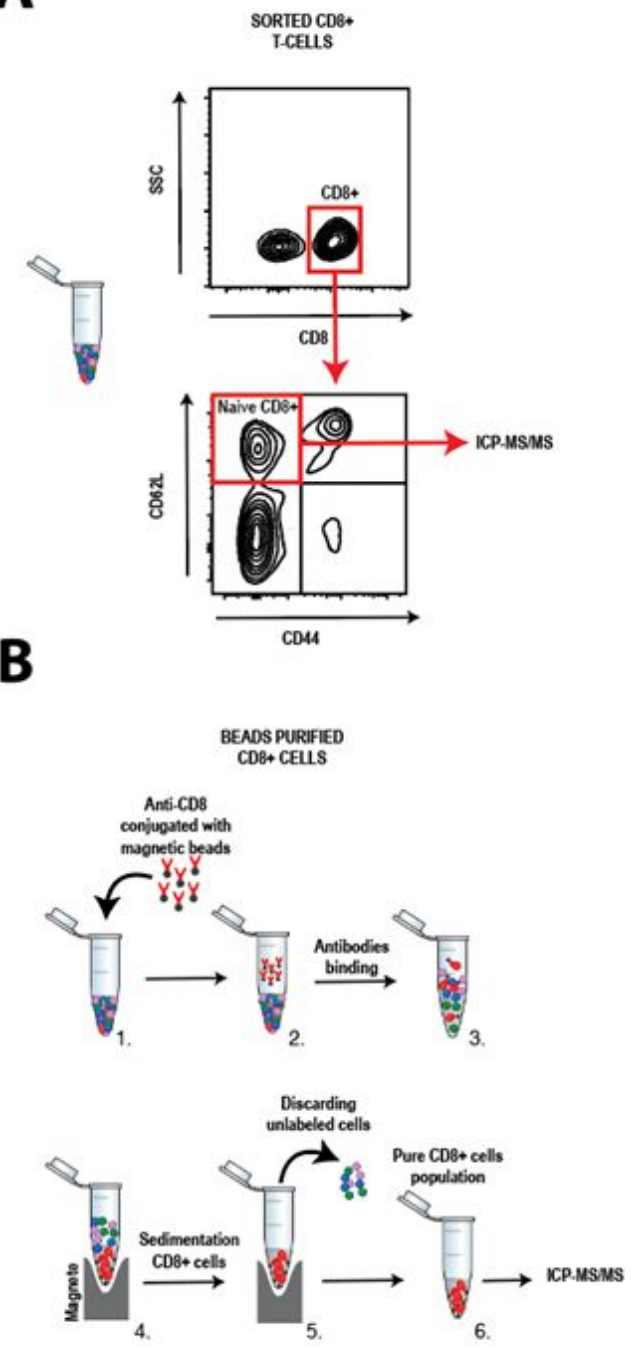

C
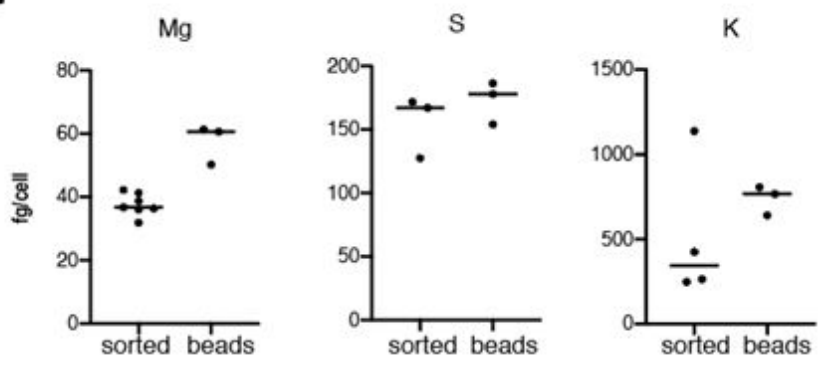

$\mathrm{Cr}$
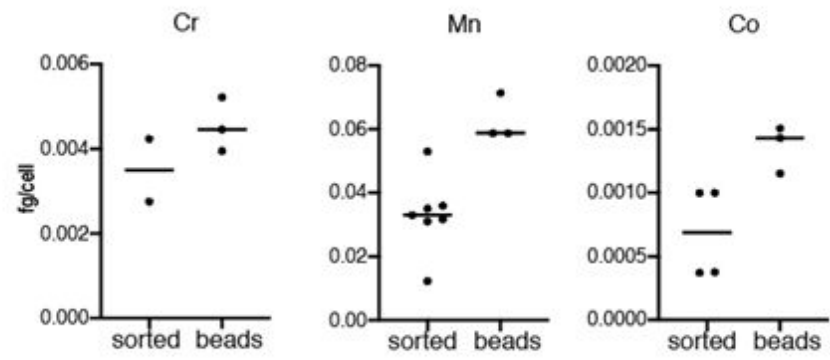

$\mathrm{Zn}$

$\mathrm{Br}$

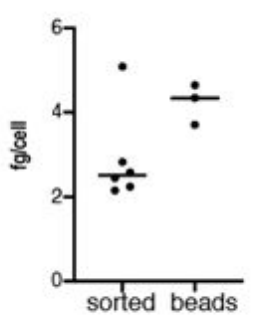

$\mathrm{Se}$

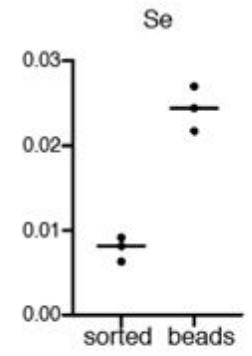

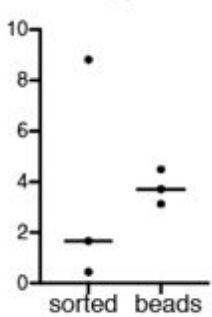

Figure S3) Comparison of elemental concentrations in T cells purified by FACS sorting and beads precipitation. A) Sorting strategy for naïve T cells based on the markers CD8, CD62L and CD44. B) Description of purification steps with anti CD8 antibody conjugated with magnetic beads. C) Mineral analyses comparison between the 2 purification methods (sorted vs beads). 